\title{
Fine-mapping of $q G W 4.05$, a major QTL for kernel weight and size in maize
}

\author{
Lin Chen, Yong-xiang Li, Chunhui Li, Xun Wu, Weiwei Qin, Xin Li, Fuchao Jiao, Xiaojing Zhang, Dengfeng Zhang, \\ Yunsu Shi, Yanchun Song, Yu Li $i^{*}$ and Tianyu Wang
}

\begin{abstract}
Background: Kernel weight and size are important components of grain yield in cereals. Although some information is available concerning the map positions of quantitative trait loci (QTL) for kernel weight and size in maize, little is known about the molecular mechanisms of these QTLs. qGW4.05 is a major QTL that is associated with kernel weight and size in maize. We combined linkage analysis and association mapping to fine-map and identify candidate gene(s) at qGW4.05.

Results: QTL qGW4.05 was fine-mapped to a 279.6-kb interval in a segregating population derived from a cross of Huangzaosi with LV28. By combining the results of regional association mapping and linkage analysis, we identified GRMZM2G039934 as a candidate gene responsible for qGW4.05. Candidate gene-based association mapping was conducted using a panel of 184 inbred lines with variable kernel weights and kernel sizes. Six polymorphic sites in the gene GRMZM2G039934 were significantly associated with kernel weight and kernel size.

Conclusion: The results of linkage analysis and association mapping revealed that GRMZM2G039934 is the most likely candidate gene for qGW4.05. These results will improve our understanding of the genetic architecture and molecular mechanisms underlying kernel development in maize.
\end{abstract}

Keywords: Maize, Kernel weight, Kernel size, Fine-mapping, Association mapping

\section{Background}

The corn kernel serves as a storage organ for assimilation products. Its yield directly influences food security. In agricultural production, maize yield is mainly composed of effective ear number, kernel number per ear and kernel weight. Kernel weight is the integrated embodiment of three elements: kernel length, kernel width and kernel thickness. Thus, understanding the genetic and molecular basis of kernel weight and kernel size is extremely important for the breeding of high-yield maize.

Due to the rapid development of molecular biotechnology, comparative genomics, and bioinformatics, many genes associated with maize flowering time, plant architecture and other traits, such as vgt1 [1], ZmCCT [2, 3], spil [4], ZmCLA4 [5], Fea2 [6, 7] and tga1 [8], have been positionally cloned. However, genes directly related to

\footnotetext{
*Correspondence: liyu03@caas.cn; wangtianyu@263.net

Institute of Crop Science, Chinese Academy of Agricultural Sciences, National Key Facility for Crop Gene Resources and Genetic Improvement (NFCRI), Beijing 100081, China
}

kernel yield are rarely identified by natural genetic variation. Most genes associated with kernel yield are isolated by making use of maize mutants, such as $g \ln 1-3$, gln1-4, rgf1, sh1, sh2, dek1, and incw2 [9-13]. These genes identified by mutant analysis have facilitated the characterization of kernel development and its regulation. However, the genetic architecture and molecular mechanisms underlying natural quantitative variation in kernel yield have not been completely elucidated.

The genetic basis of quantitative traits can be recognized more clearly through QTL mapping. Many QTLs related to kernel traits have been identified in the maize genome [14-18], but few have been positionally cloned because 1) the maize genome is large and has many transposable elements and repetitive sequences [19-23] and 2) most complex traits such as kernel yield and kernel size are controlled by many genes with small effects [24-29]. QTLs identified in different genetic backgrounds across multiple environments have a higher chance of being positionally cloned. A QTL cluster on bin 4.05 of the maize genome has been 
repeatedly associated with kernel size and weight in different populations in previous studies. Doebley et al. (1994) identified a major QTL for kernel weight in BNL5.46 - UMC42A and UMC42A - UMC66 on bin 4.05 that explained 12.82 and $15.71 \%$ of the phenotypic variance in two $F_{2}$ populations developed from maize and teosinte, respectively [30]. Ajnone-Marsan $\mathrm{P}$ et al. (1995) identified a QTL associated with grain yield on bin 4.05 using the $F_{2}$ population from a cross of B73 and A7 [31]. Peng et al. (2011) identified a QTL conferring kernel size and weight on bin 4.04-4.05 of the maize genome using two $F_{2: 3}$ populations [32]. These results demonstrate the importance of bin 4.05 for kernel size and weight and provide a target region for fine-mapping and positional cloning.

We previously identified a QTL cluster designated $q G W 4.05$ that is associated with kernel-related traits on bin 4.05 in the maize genome in different recombinant inbred line (RIL) populations across multiple environments [33]. The greatest effect of qGW4.05 on kernel weight, kernel length and kernel width (23.94, 21.39 and $10.82 \%$, respectively) was observed in the RIL population of LV28 $\times$ HZS. These effects imply that this region carries a pleiotropic gene or several closely linked genes that affect both kernel size and weight. In this study, we used the excellent inbred line Huangzaosi (HZS) which plays an important role in Chinese maize breeding and has more than 70 inbred progeny lines and 80 important hybrids [34] and the RIL families from the cross of LV28 and HZS to develop a new mapping population. Then, we combined linkage analysis and regional association mapping to 1) re-evaluate the genetic effect of $q G W 4.05$ in the new population; 2) fine-map $q G W 4.05$; and 3) infer potential candidate genes responsible for $q G W 4.05$.

\section{Results \\ Confirmation of $q$ GW4.05}

HZS and LV28 are elite inbred lines in Chinese maize breeding. HZS has a higher hundred kernel weight (21.30 g) than LV28 (18.10 g), a shorter 10-kernel length $(8.20 \mathrm{~cm})$ than LV28 $(9.40 \mathrm{~cm})$ and a wider 10-kernel width $(7.40 \mathrm{~cm})$ than LV28 $(6.30 \mathrm{~cm})$ (Fig. 1). To confirm the QTL on bin 4.05, we developed 20 new polymorphic markers (Additional file 1: Table S1) between LV28 and HZS on chromosome 4 and identified the genotype of all RIL families from LV $28 \times$ HZS. Subsequent re-mapping of $q$ GW4.05 to the interval bnlg490 umc1511 on bin 4.05 explained 23.61, 20.52, and $10.0 \%$ of the phenotypic variance in hundred kernel weight (HKW), 10-kernel length (10KL) and 10-kernel width $(10 \mathrm{KW})$, respectively (Fig. 2, Table 1$)$. Using a flanking marker of $q G W 4.05$ to screen all RIL families, we determined that those RIL families harbouring the qGW4.05HZS allele have greater kernel weight and longer and wider kernels than those harbouring the qGW4.05-LV28 allele (Fig. 1). This result is consistent with previous work [33] and indicates that qGW4.05-HZS plays a positive role in producing a larger kernel.

Subsequently, we crossed the RIL family of G184, which harbours the $q G W 4.05$ allele from LV28, with HZS to produce an RIL- $\mathrm{F}_{2}$ population. Using these $1333 \mathrm{~F}_{2}$ plants in 2012, qGW4.05 was mapped to the UMC2061-BNLG1217 interval (Additional file 1: Table S1). The allele of HZS displays partial dominance over the allele of LV28. The locus $q G W 4.05$ explained $5.17,3.01$, and $2.98 \%$ of the

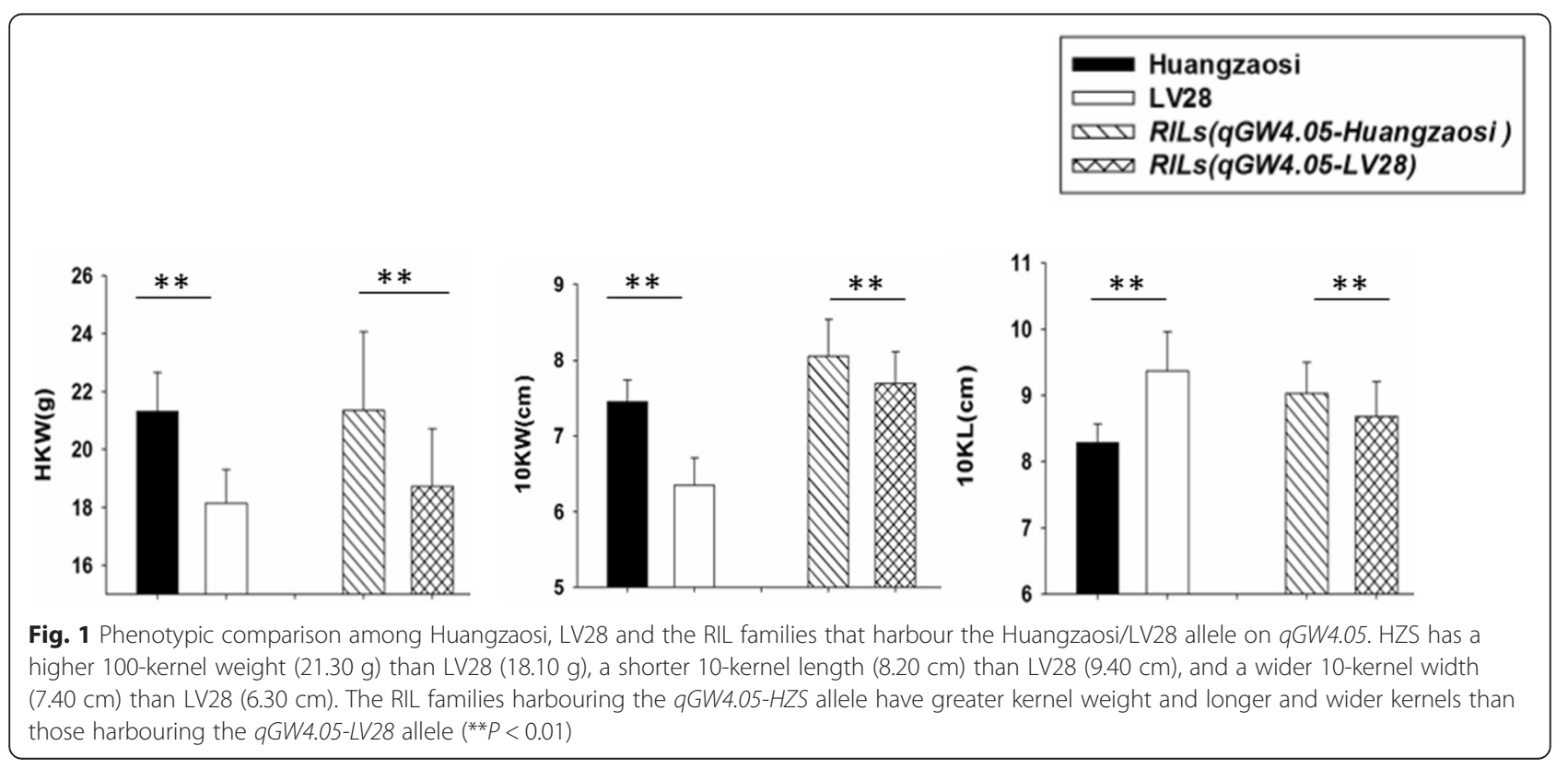




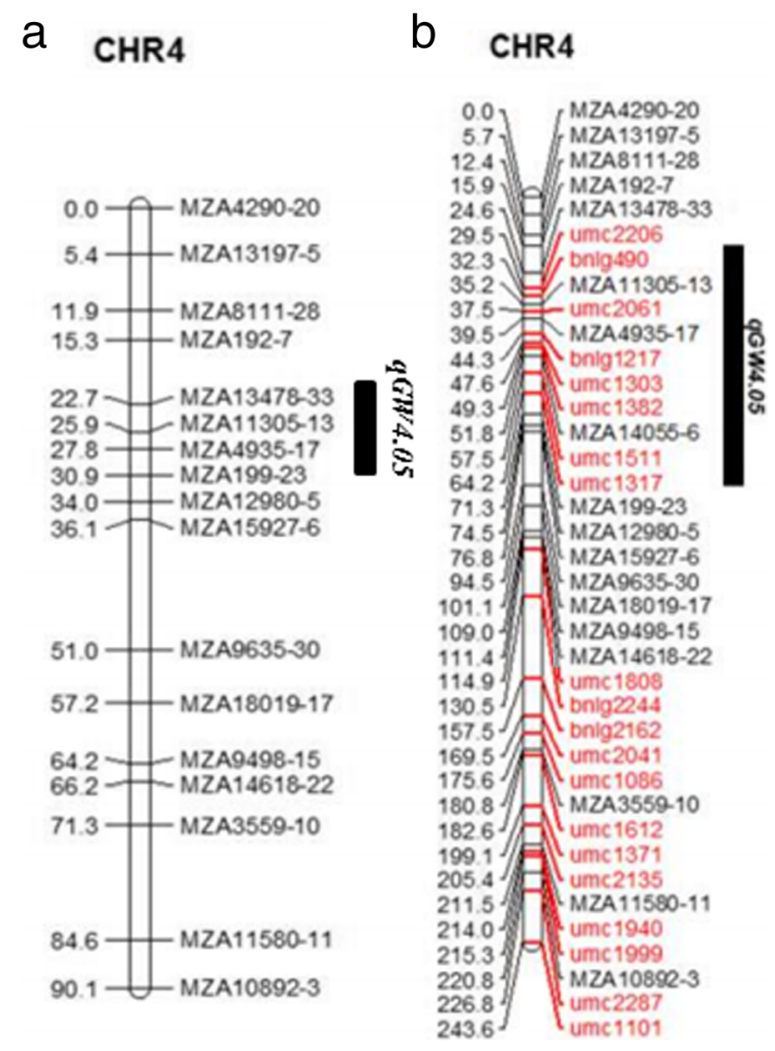

Fig. 2 The location of qGW4.05 on the different genetic maps. a The genetic map constructed in 2013 and $\mathbf{b}$ the new genetic map constructed in this study. qGW4.05 was located at MZA13478-33MZA4935-17 in 2013, and it was re-mapped in the bnlg490-umc1511 region in this study

phenotypic variance in kernel length, kernel width and kernel weight, respectively (Additional file 2: Table S2). These results confirmed that the UMC2061-BNLG1217 interval contains a functional unit controlling kernel size and weight in maize.

\section{Fine-mapping of $q G W 4.05$}

To improve the accuracy of the fine-mapping, we developed Indel (insertion and deletion) markers to replace the initial simple sequence repeat (SSR) markers; the initial SSR markers have a fuzzy physical location around qGW4.05 on chromosome 4 (30-40 Mb) of the maize genome (Additional file 1: Table S1). Using the new Indel markers to genotype the RIL- $F_{2}$ population, $q$ GW4.05 was further mapped to the ND16-ND19 interval by QTL analysis (Fig. 3a). qGW4.05 explained 7.70, 8.88 , and $7.34 \%$ of the phenotypic variance in kernel length, kernel width and kernel weight, respectively, according to the results of the re-analysis (Table 2). This result is consistent with the QTL mapping using the initial SSR markers, indicating that the physical locations of these markers are the same. We then identified five recombinant types using the new markers on the 1332 $\mathrm{F}_{2}$ individuals in 2012, among which F2-Rec1 to F2-Rec2 carried the LV28 allele in the ND16-ND19 interval, whereas F2-Rec3 to F2-Rec5 carried the HZS allele in the corresponding interval (Fig. 3b). The 100-kernel weight of F2-Rec1 to F2-Rec2 was distinctly less than that of heterozygotes in this region and less than that of F2-Rec3 to F2-Rec5 (Fig. 3b), indicating that the ND16ND19 interval may contain a QTL for kernel weight. Similar performance in kernel length and kernel width was observed (Fig. 3b), suggesting that the ND16-ND19 interval might contain a pleiotropic QTL.

A larger segregating population with $8000 \mathrm{~F}_{3}$ individuals was developed from the $F_{2}$ plants, which are heterozygous in the ND16-ND19 interval, and used to fine-map $q G W 4.05$ in summer 2013. Furthermore, new markers were developed to identify recombinants in the ND16-ND19 interval. Using the same analytical method, we successfully narrowed qGW4.05 to the NO4-ND4M26 interval in the maize genome, which is $279.6 \mathrm{~kb}$ long (Fig. 3c). There was no significant difference in kernel weight between LV28 and F3-Rec3 to F3-Rec5 carrying the LV28 allele in the NO4-ND4M26 interval on the maize genome (Fig. 3c). In addition, the kernel weight of F3-Rec1 to F3-Rec2 carrying the HZS allele in the NO4-ND4M26 interval was greater than that of LV28 (Fig. 3c). The kernel width of F3-Rec1 to F3-Rec2 was greater than that of LV28, and F3-Rec3 to

Table 1 QTLs detected in the different linkage map

\begin{tabular}{|c|c|c|c|c|c|c|c|}
\hline Linkage map & Trait & Chromosome & Position $(\mathrm{cM})^{\mathrm{a}}$ & Marker interval ${ }^{b}$ & $\operatorname{LOD}^{c}$ & PVE $(\%)^{d}$ & Add $^{\mathrm{e}}$ \\
\hline \multirow[t]{3}{*}{ Linkage map 2011} & HKW & 4 & 26 & MZA11305-13 - MZA4935-17 & 10.86 & 23.94 & -1.32 \\
\hline & $10 \mathrm{KL}$ & 4 & 25 & MZA13478-33 - MZA11305-13 & 10.06 & 21.39 & -0.24 \\
\hline & $10 \mathrm{KW}$ & 4 & 26 & MZA11305-13 - MZA4935-17 & 5.08 & 10.82 & -0.15 \\
\hline \multirow[t]{3}{*}{ New linkage map 2012} & HKW & 4 & 36 & MZA11305-13-umc2061 & 10.30 & 23.60 & -1.31 \\
\hline & $10 \mathrm{KL}$ & 4 & 34 & bnlg490-MZA11305-13 & 9.82 & 20.51 & -0.23 \\
\hline & $10 \mathrm{KW}$ & 4 & 52 & MZA14055-6-umc1511 & 4.78 & 9.97 & -0.15 \\
\hline
\end{tabular}

Notes: Position ${ }^{a}$, the genetic location of the QTL; Marker interval ${ }^{b}$, the flanking marker interval of the QTL; LOD ${ }^{c}$, Logarithm of odds for each QTL; PVE (\%) ${ }^{\text {, }}$ percentage of phenotypic variance explained by a QTL; $\mathrm{A}^{\mathrm{e}}$, additive values (a positive value indicates that the additive effect was derived from LV28, and a negative value indicates derivation from Huangzaosi) 


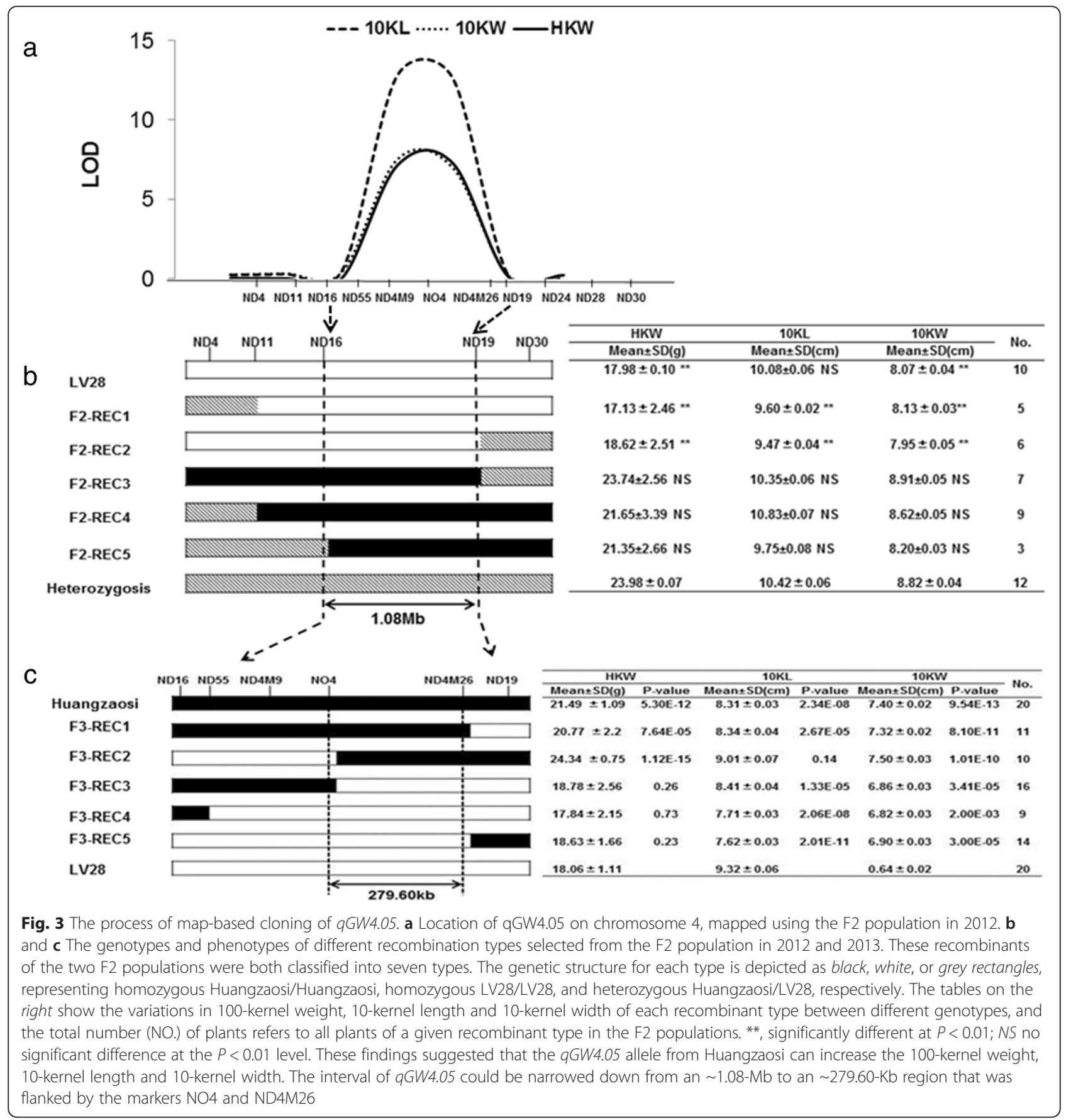

Table 2 aGW4.05 location in the F2 population in 2012

\begin{tabular}{lllcccc}
\hline Trait & Chromosome & Marker interval & LOD $^{\mathrm{b}}$ & PVE (\%) & Add $^{\mathrm{d}}$ & Dom $^{\mathrm{e}}$ \\
\hline 10KL & 4 & ND16-ND19 & 8.63 & 7.70 & -0.03 & 0.01 \\
10KW & 4 & ND16-ND19 & 9.93 & 8.88 & -0.03 & 0.01 \\
HKW & 4 & ND16-ND19 & 8.12 & 7.33 & -2.30 & 0.48
\end{tabular}

Notes: Marker interval ${ }^{a}$, the flanking marker interval of the QTL; LOD ${ }^{b}$, Logarithm of odds for each QTL; PVE (\%) ${ }^{c}$, percentage of phenotypic variance explained by a QTL; $A^{d}$, additive values (a positive value indicates that the additive effect was derived from LV28, and a negative value indicates derivation from Huangzaosi); $D^{e}$, dominant values
F3-Rec5 carrying the LV28 allele in the interval were closer to LV28 than were F3-Rec1 and F3-Rec2 carrying the HZS allele of $q G W 4.05$. However, the kernel length was the same between F3-Rec1 to F3-Rec3 and F3-Rec4 to F3-Rec5 (Fig. 3c). The unexpected kernel size performance can be attributed to the strong environmental influence on kernel-related traits. In conclusion, we confirmed that there is a gene controlling kernel weight that also likely affects kernel length and kernel width in specific environments. 
Validation of $q G W 4.05$ in the RIL population

We next determined whether the restricted interval (NO4-ND4M26) is present in the RIL population from the cross of HZS and LV28 and has significant genetic effects on phenotypes. Kernel weight and kernel size were evaluated in six different environments [33]. We used the markers NO4 and ND4M26 to genotype the RIL population. Among the RILs, 68 and 79 families were homozygous for HZS and LV28, respectively. Kernel weight and kernel width differed significantly $(P<0.01)$ between the RILs homozygous for HZS and LV28 in all six environments (Fig. 4, Additional file 3: Figure S1), and kernel length differed significantly $(P<0.01)$ in all but the Xinjiang 2010 environment (Additional file 4: Figure S2). These findings suggest that the QTL in the interval of NO4-ND4M26 can affect kernel weight and kernel size in the RIL population, which is in agreement with our previous fine-mapping results.

\section{Regional association mapping}

We used the strategy of regional association mapping to further narrow down $q G W 4.05$ and identify candidate genes. An association mapping panel that contains 541 inbreed lines was field evaluated at three locations in 2 years. We selected single-nucleotide polymorphisms (SNP) markers in an interval (30-40 Mb) containing the sequence of UMC2061-BNLG1217 on chr4 of the maize genome. Using the mixed linear model, we identified one SNP, SYN4401, that was associated with the variation in kernel weight and 10-kernel width and explained 6.31 and $4.76 \%$ of the phenotypic variation in kernel weight and kernel width, respectively (Fig. 5). However, no marker was identified that was significantly associated with kernel length.

\section{Prediction of candidate genes}

The NO4-ND4M26 interval on the B73 genome is $279.6 \mathrm{~kb}$ long and contains only two genes (GRMZM2G702403 and GRMZM2G039934) and some transposable elements annotated in B73 reference genome v2.0 assembly (B73 RefGen_v2). Previous studies have demonstrated that GRMZM2G702403 is not expressed in developing kernels [35, 36]. The SNP SYN4401, which was identified by regional association mapping, is located in the gene GRMZM2G039934. We therefore considered this gene a candidate gene controlling kernel weight and size. GRMZM2G039934 encodes a putative leucine-rich repeat receptor-like protein kinase family protein. Sequencing revealed 18 SNPs and one Indel in the exons of this gene between HZS and LV28. These variations in the coding region cause eight amino acid substitutions (Table 3). SIFT
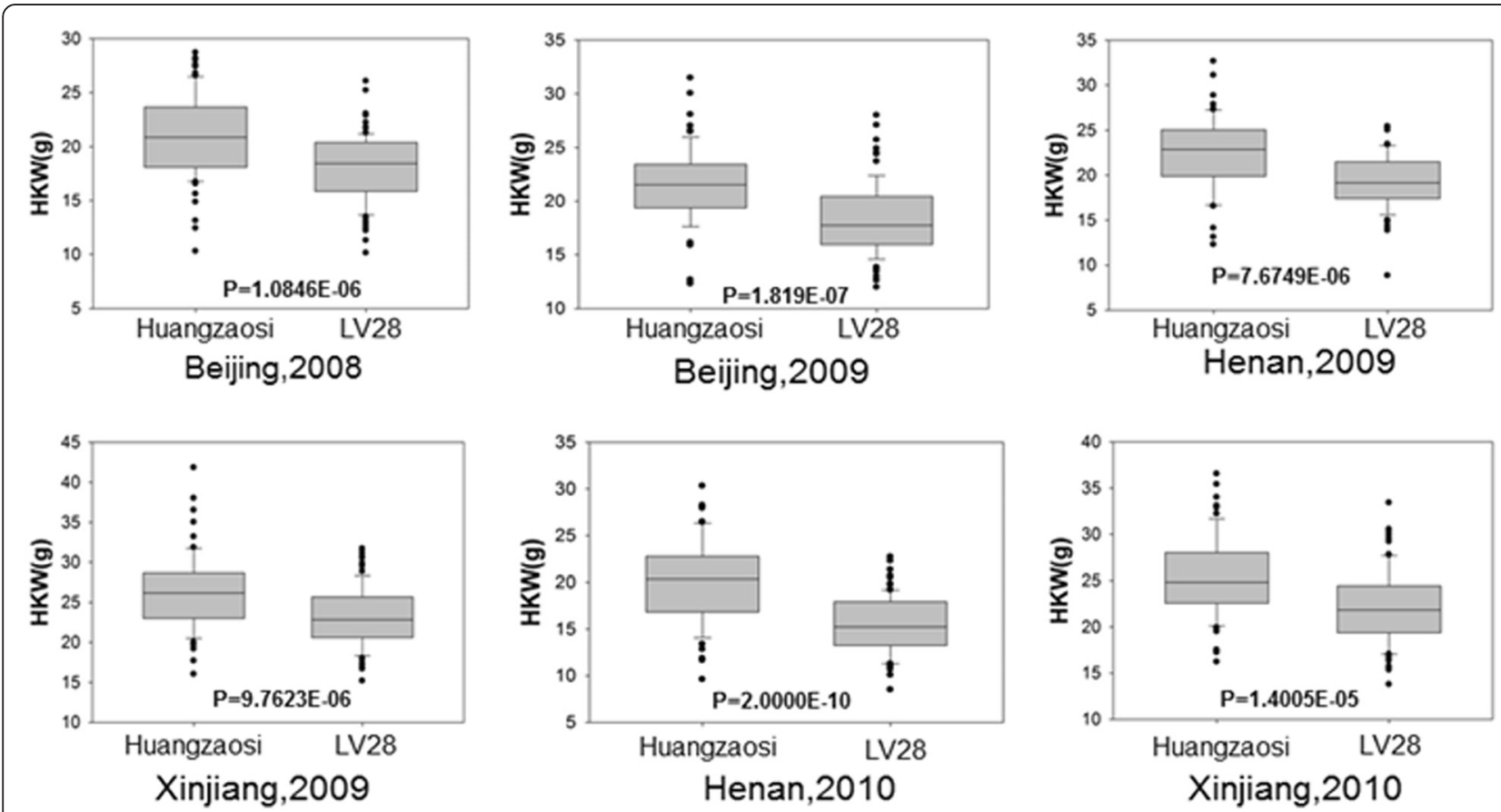

Fig. 4 Validation of qGW4.05 for hundred kernel weight (HKW) in the RIL population in six different environments. The RILs were genotyped by using the markers NO4 and ND4M26. The distributions and mean values for HKW are shown for the two homozygous genotypes, Huangzaosi and LV28, at six experimental sites. Compared with the RIL families with the LV28 homozygous genotype at the qGW4.05 region, the RIL families with the Huangzaosi homozygous genotype at the qGW4.05 region had significantly higher $(P<0.01)$ hundred-kernel weight across the six different environments 


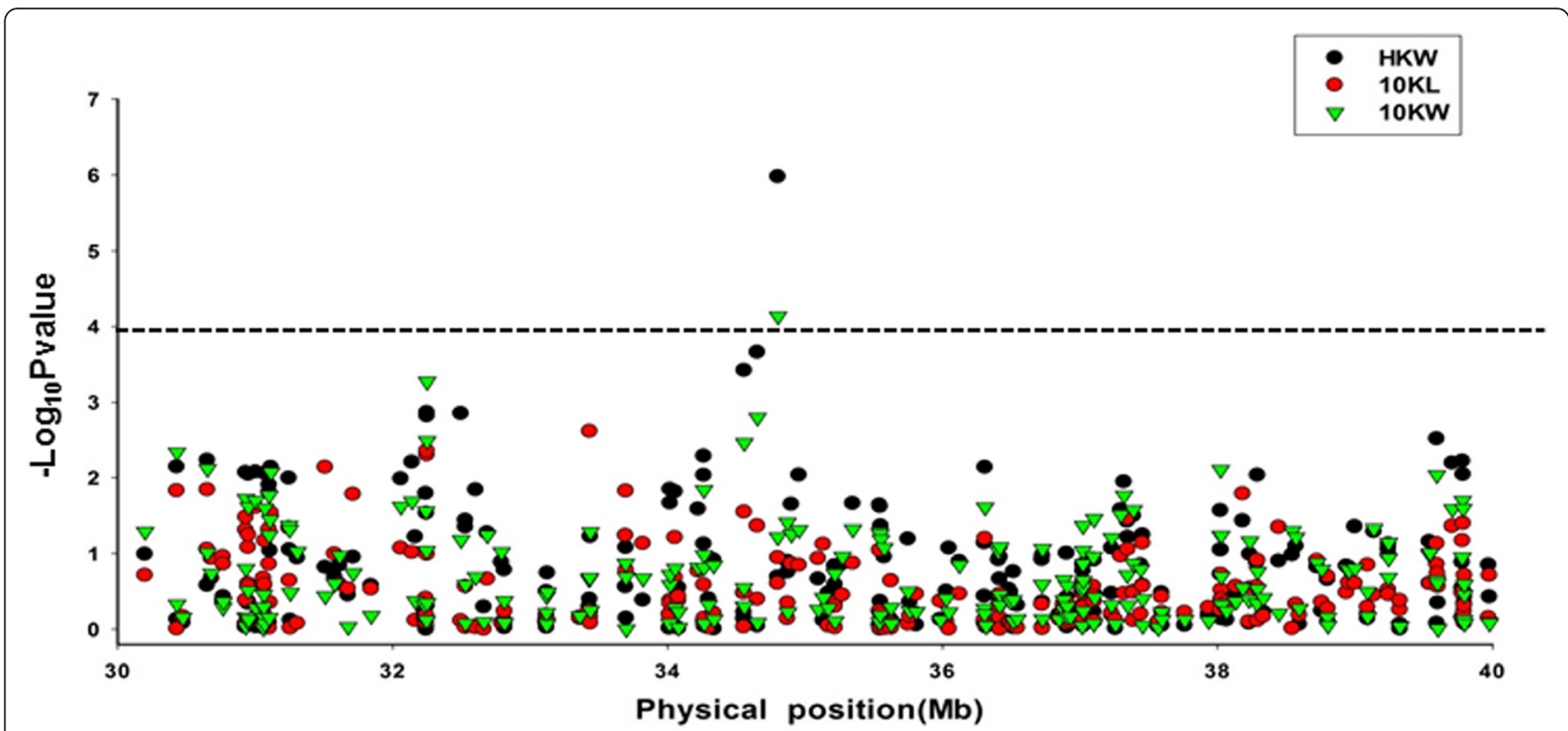

Fig. 5 Results of regional association mapping. MLM tests at the region 30-40 Mb of chromosome 4. Only SYN4401 was significantly associated with 100-kernel weight and 10-kernel width (LOD>4)

analysis, which assesses whether an amino acid substitution affects the structure of a protein or its function, revealed that one of the eight substitutions was predicted with high confidence to result in the loss of protein function of GRMZM2G039934 (Table 3). The threonine encoded by the HZS allele is hydrophilic, whereas the isoleucine encoded by the LV28 allele is hydrophobic. This amino acid substitution may result in different protein functions that underlie the differences in 100-kernel weight and kernel size between HZS and LV28.

Table 3 Polymorphic sites causing amino acid changes in the protein of GRMZM2G039934

\begin{tabular}{|c|c|c|c|}
\hline Gene ID & $\begin{array}{l}\text { Amino acid substitution } \\
\text { (Huangzaosi/LV28) }^{\mathrm{a}}\end{array}$ & $\begin{array}{l}\text { PROVEAN } \\
\text { score }^{\text {b }}\end{array}$ & $\begin{array}{l}\text { Prediction } \\
(\text { cutoff }=-2.5)^{c}\end{array}$ \\
\hline \multirow[t]{8}{*}{ GRMZM2G039934 } & $\mathrm{R} 42 \mathrm{C}$ & -0.43 & Neutral \\
\hline & T55। & -3.72 & Deleterious \\
\hline & Q371R & -0.82 & Neutral \\
\hline & I375L & 0.16 & Neutral \\
\hline & M380V & 0.26 & Neutral \\
\hline & N386K & -0.09 & Neutral \\
\hline & K387I & -1.16 & Neutral \\
\hline & D388N & -0.65 & Neutral \\
\hline
\end{tabular}

Notes: a Amino acid substitution format is $X \# Y$, where $X$ is the original amino acid, \# is the position of the substitution, and $Y$ is the new amino acid. ${ }^{b} A$ delta alignment score is computed for each supporting sequence. The scores are then averaged within and across clusters to generate the final PROVEAN score. If the PROVEAN score is equal to or below a predefined threshold (e.g. -2.5 ), the protein variant is predicted to have a "deleterious" effect. If the PROVEAN score is above the threshold, the variant is predicted to have a "neutral" effect; ' for maximum separation of the deleterious and neutral protein variants, the default score threshold is currently set at -2.5 for binary classification

\section{Association mapping of the candidate gene and} haplotype analysis

To determine the sites responsible for the differences in kernel size and kernel weight between HZS and LV28, the allelic variations of 19 sequence polymorphisms (Additional file 5: Figure S3) identified in HZS and LV28 were exclusively analysed in 184 inbred maize lines. The alleles in each polymorphic site with minor allele frequency $>0.05$ were used for association mapping using the mixed linear model (MLM), controlling for population structure $(\mathrm{Q})$ and kinship (K) (MLM Q+K). The results revealed that one polymorphism (S453) in the coding region and two polymorphisms (S881and S891) in the intron were associated with kernel length, three polymorphisms (S527, S782 and S1031) in the coding region were associated with kernel width, and two polymorphisms (S782 and S1031) in the coding region were associated with kernel weight at the $P<0.01$ level (Fig. 6). However, none of these polymorphisms generates an amino acid substitution.

Haplotype analysis suggested that S453, S881 and S891, which are associated with kernel length, might classify the population into two types. The two haplotypes differed significantly in kernel length at the $P<0.05$ level (Fig. 7), but both the HZS and LV28 alleles belong to haplotype 2 . S527, S782 and S1031, which are significantly associated with kernel width, may divide the panel into four haplotypes. The phenotypes of haplotype 1 , haplotype 2 and haplotype 3 did not differ significantly but were significantly wider than haplotype 4 (Fig. 7). The kernel width for haplotype 1, which corresponds to the HZS genotype, was significantly higher than that of haplotype 4 , which corresponds to the LV28 genotype, consistent with the 


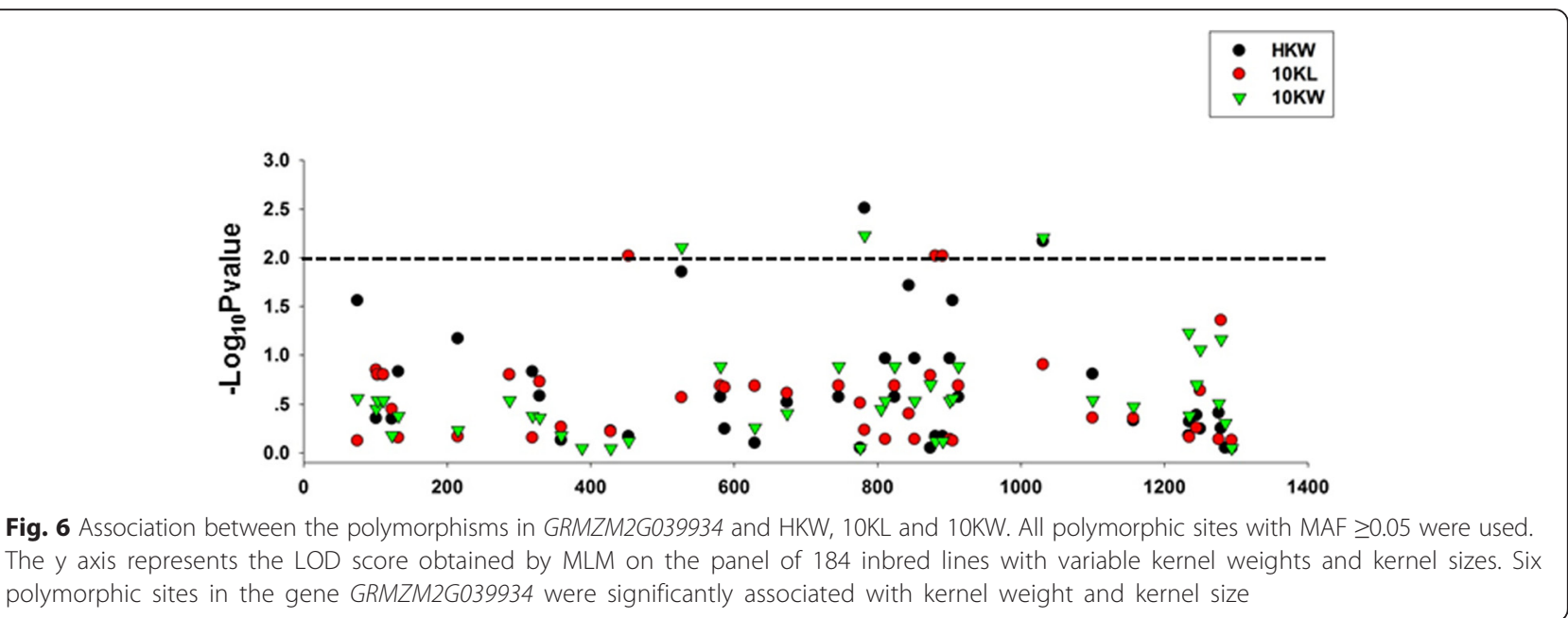

kernel width difference between HZS and LV28. S782 and S1031, which are related to 100-kernel weight, form three different haplotypes (Fig. 7). The phenotype of haplotype 3 , which corresponds to the LV28 genotype, had a smaller kernel weight than those of haplotypes 1 , and haplotype 2 which corresponds to the HZS genotype.

\section{Discussion}

Comparison of $q G W 4.05$ and other major QTL for kernel weight and size

Kernel weight and size, as yield components, are typical quantitative traits that are controlled by multiple genes and sensitive to environmental impacts. The

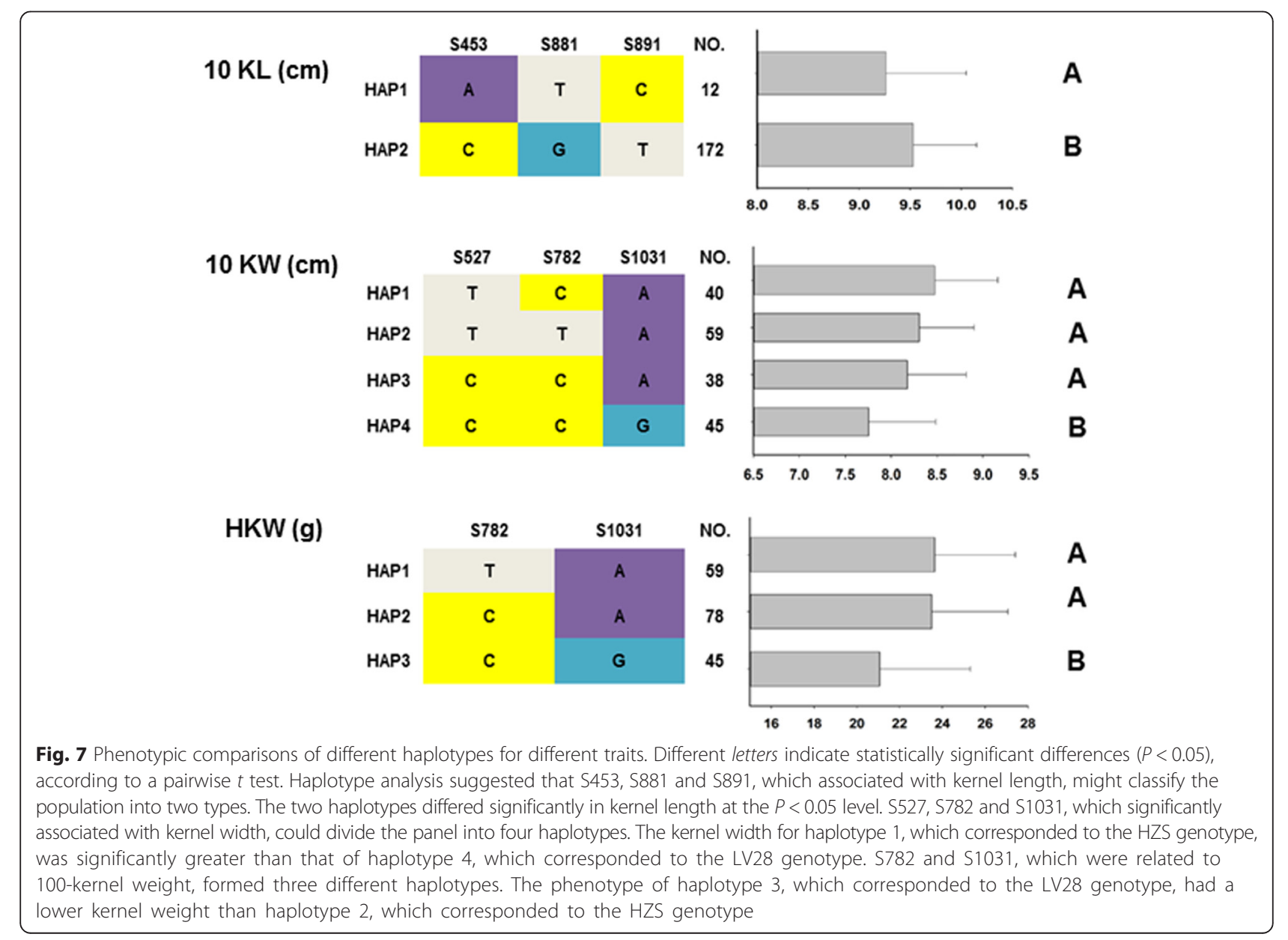


development of molecular markers has led to the identification of 200 QTL related to kernel weight and size distributed in the entire genome according to data in the MaizeGDB (http://www.maizegdb.org). In bin4.05, multiple QTL associated with yield components have been found: qcobd8 for cob diameter [37], qgyld12 for grain yield [31], qkrow7 for kernel row number [37] and $q k w 24$ for kernel weight [30]. Peng et al. (2011) identified a QTL cluster for kernel weight and kernel length in bin4.05 with two $F_{2: 3}$ populations [38]. Li et al. (2011) and Wang et al. (2013) both identified a metaQTL associated with yield components by meta-analysis in bin4.05 [39, 40]. These results implied that $q G W 4.05$ with these QTL formed a core cluster for QTL controlling different kernel related traits.

Prado et al. (2014) have found multiple QTL related to kernel weight, located in bins 1.01, 1.05, 1.11, 3.06, 5.05, 9.05 and 10.03 [41]. Liu et al. (2014) identified 6, 16 and 15 QTL related to kernel length, kernel width and kernel weight, respectively [16]. Zhang et al. (2014) found 42 main-effect QTL related kernel weight and size [14]. Only a few of these QTL can be found in different genetic background and different environments. Among these QTL, digenic interactions involving multiple loci over the whole genome have been shown to be related to kernel weight and size. Like these QTL, qGW4.05 can explain $23.94,21.39$ and $10.82 \%$ of the phenotypic variance in hundred-kernel weight, 10-kernel length and 10kernel width, respectively. Compared with the above QTL, qGW4.05 can be found in many different populations including the $F_{2}$ populations from the cross of maize and teosinte [30], the $F_{2}$ population from a cross of B73 and $A 7$ [31], the $F_{2: 3}$ populations from Huangzaosi and Qi319, the RIL population from Huangzaosi and other inbred lines [33, 38]. Based on the genetic linkage map constructed using 2091 bins as markers, we don't found the digenic interaction between $q G W 4.05$ and other quantitative trait loci (data unpublished). These results suggested that the genetic bases of kernel weight and size are very complex and that positional cloning of these QTL will be very difficult. Compared with these QTL, $q$ GW4.05 may allow more efficient positional cloning of the candidate gene.

\section{qGW4.05 is an important and pleiotropic locus}

High-throughput SNP genotyping analysis of elite maize germplasm in China identified bin 4.05 as one of the conserved regions transmitted from Huangzaosi, an important foundation parent, to its descendants [42]. The locus $q G W 4.05$ is present across multiple environments and different genetic backgrounds such as Huangyesi3, LV28, QI319, Huobai and Duo229. Among the different populations, $q G W 4.05$ is related to multiple kernel traits.
In the above populations, $q G W 4.05$-HZS is positive for kernel-related traits, whereas other parents are negative for these traits. These results suggest that qGW4.05 is very important for HZS and HZS-derived lines and is a positive QTL for kernel-related traits.

Many previous studies have indicated that yield and kernel-related traits are controlled by a set of QTLs, some of which are QTL clusters $[9,17,18,30,32,33$, 38, 43-47]. The distribution of these QTL clusters can be explained by a pleiotropic QTL or multiple tightly linked QTLs. When a high-resolution map has been constructed, a QTL cluster can be resolved into many minor effect QTLs. QTL analysis in maize has clearly demonstrated that many complex traits controlled by QTL clusters, such as the grain yield, kernel size and other agronomic traits, can be broken down into many QTLs once the linkage map has been improved [33, 48, 49]. However, a QTL cluster may contain only one major QTL that controls multiple related traits and thus has pleiotropic effects. In the present study, QTL mapping in the RIL families restricted $q G W 4.05$ to a $10-\mathrm{Mb}$ interval and revealed its relationship to both kernel size and kernel weight. When the interval was further narrowed to $1 \mathrm{Mb}, q G W 4.05$ remained associated with the three traits. This finding suggests that qGW4.05 may be a pleiotropic locus that affects kernel size and kernel weight in maize.

\section{GRMZM2G039934 is involved in the development of maize kernels via a different mechanism than in rice} In this study, we successfully fine-mapped $q G W 4.05$ to a $297.2 \mathrm{~kb}$ interval. Previous studies have indicated that only GRMZM2G039934 is expressed in this interval in kernels of maize $[18,19]$. Regional association mapping revealed that the SNP SYN4401, which is located in GRMZM2G039934, is significantly associated with 100-kernel weight and 10-kernel width. We therefore propose that GRMZM2G039934 is a candidate gene related to the development of maize kernels. In rice, a 1-bp deletion in GW2 results in a premature stop codon. The loss of function of GW2 leads to an increased cell number, a wider spikelet hull and an accelerated grain milk-filling rate, which increases grain width, weight and yield [50]. Like GW2, a single SNP in exon2 of GS3 results in a premature stop codon. The shorter protein is associated with a longer grain length and larger grain weight [44]. A 1212-bp deletion in GW5 is associated with increased grain width in rice [45]. However, we did not identify any deletion or SNP changes resulting in a premature stop codon in GRMZM2G039934 in maize. Thus, the mechanisms underlying kernel development and regulation may differ between maize and rice. 
GRMZM2G039934 encodes a putative leucine-rich repeat receptor-like protein kinase family protein. The protein product of the candidate gene is in the same family as $d$ warf61, which is involved in the brassinosteroid (BR) biosynthesis network and influences grain size development in rice [51]. Studies in Arabidopsis and rice have demonstrated that brassinosteroids play an important role in seed development [51-56]. Many BR-deficient mutants of Arabidopsis (dwf5, shk1-D) and rice $(b r d 2, d w f 11, d 61)$ have a common phenotype that includes dwarfism, short organs, and small grains. Moreover, overexpression of BR biosynthesisrelated genes increases grain size and the number of grains. These results suggest that BRs play a key role in normal seed development. However, the detailed mechanisms of BR regulation of seed development remain unclear. The rice dwarf mutant $d 61$ has a phenotype of smaller grains and lower kernel weight compared to wild type due to loss of function of the rice brassinosteroid insensitive1 orthologue OsBRI1 [51]. The mutants have higher biomass than wild type under high planting density. Moreover, the partial suppression of OsBRI1 can increase grain yield by regulating the brassinosteroid biosynthesis network in transgenic rice plants. GRMZM2G039934 may be involved in the same biosynthetic process in maize. Detailed studies are necessary to reveal the mechanisms by which GRMZM2G039934 regulates kernel development in maize.

\section{qGW4.05 for maize breeding}

Maize is the most widely grown crop in the world, and to improve the grain yield has always been a top priority [57]. Identifying useful QTLs related to grain yield such as kernel weight, kernel size and kernel number is important for genetic manipulation to increase production via maize breeding. There are many successful examples of the introduction of useful QTLs. For example, the introduction of $q H S R 1$, which is a QTL related to head smut in head smut-susceptible lines via marker-assisted selection, has significantly reduce disease incidence over time in maize $[58,59]$. $q$ GW4.05 has been identified in different populations and in different environments [33]. In this study, the presence of $q G W 4.05$ was confirmed using two $F_{2}$ populations of various sizes and regional association mapping analysis in a panel of 541 inbreed lines. Therefore, $q G W 4.05$ may be utilized in maize breeding by marker-assisted selection. The LV28 allele at qGW4.05 decreases 100-kernel weight and kernel size relative to the HZS allele; thus, it may be feasible to use lines carrying the HZS allele to improve lines carrying the LV28 allele in $q G W 4.05$. In particular, the two SNP sites S782 and S1031, which are associated with kernel weight and kernel width, could help breeders to select wider and heavier kernels of maize in the future.

\section{Conclusions}

We combined linkage analysis and association mapping to fine-map and identify candidate gene(s) at qGW4.05, a major quantitative trait locus (QTL) associated with maize kernel weight and size. QTL qGW4.05 was fine-mapped to a $279.6-\mathrm{kb}$ interval in a segregating population derived from a cross of Huangzaosi with LV28. We identified GRMZM2G039934 as the candidate gene responsible for $q G W 4.05$. Furthermore, six polymorphic sites in the gene GRMZM2G039934 were significantly associated with kernel weight and size. These results will improve our understanding of the genetic architecture and molecular mechanisms underlying kernel development in maize, which are important components of grain yield.

\section{Methods}

Plant materials used for fine-mapping of qGW4.05

$q$ GW4.05 controlling 100-kernel weight and kernel size was previously mapped to bin 4.05 of chromosome 4 using the RIL population from the cross of HZS and LV28 [33]. In the present study, we used G184, an RIL family from the above cross that harbours the LV28 allele of $q$ GW4.05, to develop RIL- $\mathrm{F}_{2}$ with HZS. A total of 1332 RIL- $F_{2}$ individuals were used to confirm the accurate physical location of $q G W 4.05$. We then selected heterozygous individuals using markers flanking $q G W 4.05$ for self-pollination to develop the RIL- $F_{3}$ population. The RIL- $F_{3}$ population, which contained approximately 8000 individuals, was used to fine-map qGW4.05. Individuals containing recombination breakpoints within the QTL interval were selected from the RIL- $F_{3}$ population for self-pollination to conduct a progeny test. Moreover, an association mapping panel (AP) with 541 inbred maize lines covering a wide range of genetic variation was used for regional association mapping. All plant materials in this study were conserved in our experiment lab and we declare that all plant materials in this study comply with the "Convention on the Trade in Endangered Species of Wild Fauna and Flora'.

\section{Field design and phenotypic evaluation}

The RIL population was field evaluated previously [33]. The RIL- $\mathrm{F}_{2}$ and RIL- $\mathrm{F}_{3}$ populations were planted in summer 2012 and 2013 in Beijing $\left(39.48^{\circ} \mathrm{N}, 116.28^{\circ} \mathrm{E}\right.$, in northern China). The progeny were tested in summer 2014 in Beijing. The association panel was field evaluated for the target phenotypes in nine environments: Changchun in Jilin province in $2011\left(43.88^{\circ} \mathrm{N}, 125.35^{\circ}\right.$ $\mathrm{E}$, in northeastern China), Beijing in 2011 and 2012, Tai'an in Shandong province in 2011 and $2012\left(36.11^{\circ} \mathrm{N}\right.$, 
$117.08^{\circ} \mathrm{E}$, in eastern China), Xinxiang in Henan province in 2011 and $2012\left(30.77^{\circ} \mathrm{N}, 106.10^{\circ} \mathrm{E}\right.$, in central China), and Nanchong in Sichuan province in 2011 and $2012\left(43.88^{\circ} \mathrm{N}, 125.35^{\circ} \mathrm{E}\right.$, in southwestern China). The institute of crop science belonging to the Chinese Academy of Agricultural Sciences has set up experimental field bases at all the above locations. The institute of crop science was approved for field experiments, and the field studies did not involve endangered or protected species.

The field experiment methodology and the evaluation of kernel-related traits for the populations used in this study were identical to those described in a previous study [33]. The populations were arranged in a randomized complete block design, and each genotype was grown in a single row $3 \mathrm{~m}$ in length with $0.6 \mathrm{~m}$ between adjacent rows, with 12 individual plants per row. The field management followed normal agricultural practices. After harvest, the kernels were threshed from the middle part of the ears to determine the 100-kernel weight (HKW, g), 10-kernel width (10KW, cm) and 10-kernel length $(10 \mathrm{KL}, \mathrm{cm})$, which were estimated from the average of three measurements.

\section{Molecular marker development}

The SSRs used for the RIL population were selected from MaizeGDB (http://www.maizegdb.org). According to re-sequencing information regarding HZS and LV28 provided by Professor Jinsheng Lai of China Agricultural University [60], PCR-based Indel markers and sequencebased SNP markers in the interval of the qGW4.05 region were designed using Primer Premier 5.0 (PREMIER Biosoft International, USA) with a product size $<300 \mathrm{bp}$. All markers are listed in Table 1 and were used to identify the genotype of the RIL- $\mathrm{F}_{2}$ and RIL- $\mathrm{F}_{3}$ populations. Of 56,110 SNPs derived from the MaizeSNP50 BeadChip within the confidence interval of $q G W 4.05,256$ SNPs were selected for association analysis of the association mapping panel (AP).

\section{Genotyping and QTL analysis}

Genomic DNA was extracted from fresh maize seedling leaves using the cetyltrimethylammonium bromide (CTAB) method [61]. A marker linkage map was constructed using the Kosambi function of MAPMAKER/ EXP version 3.0 [62]. A mixed model based on the composite interval mapping method was used to conduct QTL analysis by QTL IciMapping V3.3 [63, 64]. The threshold for indicating the existence of a significant QTL for 100-kernel weight and kernel size in each generation was obtained by 1000 permutations at a significance level of $P=0.05$. The significance of the phenotypic differences for different recombinant types relative to LV28 or heterozygosis was evaluated using Student's $t$ test in SAS (SAS Institute, Inc., Cary, NC).

\section{Regional association mapping}

Both the kinship matrix and the principal component analysis (PCA) were calculated using allelic data from 4544 SNP markers of 56,110 derived from the MaizeSNP50 BeadChip that were evenly distributed across the whole maize genome. Alleles of each polymorphism with minor frequency $>0.05$ were used for association mapping using the mixed linear model (MLM) controlling for population structure (Q) and kinship (K) (MLM Q+K). Significant marker-trait associations were declared for LOD $>4$. All associations were analysed with TASSEL5.0 [65, 66]. LD analysis within the target region was performed using the software Haploview [67].

\section{Candidate gene sequencing and association mapping}

The genomic DNA sequences of candidate genes from HZS and LV28 were obtained by polymerase chain reaction (PCR) amplification using the primers N37F and N37R. PCR was performed using high-fidelity LA Taq Mix (Takara, http://www.clontech.com/takara). The purified PCR products were cloned into pLBVector (TIANGEN, http://www.tiangen.com) according to the manufacturer's instructions. Three positive clones were sequenced for each sample. Sequence contig assembly and alignment were performed using DNAMAN version 5.2.2 (LynnonBiosoft, http://www.lynnon.com).

A subset of 184 inbred lines from the regional association mapping panel were used for candidate gene-based association mapping. The primers N37F/R were used to amplify the candidate gene's coding region. The PCR products of three repetitions were directly sequenced. Initial alignment and manual refinement of the alignment were performed using BioEdit software [68]. Sites with allelic frequency $>0.05$ were used for subsequent analysis. Association mapping was performed with TASSEL 2.1 using an MLM Q+K model $[65,66]$.

\section{Ethics}

The experiments comply with the ethical standards in the country in which they were performed.

\section{Consent to publish \\ Not applicable.}

\section{Availability of data and materials}

The data supporting the results of this article are included within the article and its additional files. The candidate gene (GRMZM2G039934) sequences of Huangzaosi and LV28 were deposited in the Genbank (https://www.ncbi.nlm.nih.gov/genbank) under accession number KU933938 and KU933939, respectively. 


\section{Additional files}

Additional file 1: Table S1. The primers used in this study for finemapping qGW4.05 and candidate gene sequencing. (XLS $30 \mathrm{~kb}$ )

Additional file 2: Table S2. qGW4.05 location in the RIL-F2 population in 2012 using the SSR markers. (DOCX 16 kb)

Additional file 3: Figure S1. Validation of aGW4.05 for 10KW in the RIL population in six different environments. The RILs were genotyped by using the markers NO4 and ND4M26. The distributions and mean values for $10 \mathrm{KW}$ are shown for the two homozygous genotypes, Huangzaosi and LV28, at the six experimental sites. Across the six environments, the RIL families that had the Huangzaosi homozygous genotype at the qGW4.05 region had significantly wider kernels $(P<0.01)$ than the families that had the LV28 homozygous genotype. (TIF $112 \mathrm{~kb}$ )

Additional file 4: Figure S2. Validation of qGW4.05 for 10KL in the RIL population in six different environments. The RILs were genotyped by using the markers NO4 and ND4M26. The distributions and mean values for $10 \mathrm{KL}$ are shown for the two homozygous genotypes, Huangzaosi and LV28, at six experimental sites. Across all of the environments except Xinjiang-2010, the RIL families harbouring the qGW4.05-Huangzaosi allele had significantly longer kernels $(P<0.01)$ than the families harbouring the qGW4.05-LV28 allele. (TIF 99 kb)

Additional file 5: Figure S3. DNA sequence alignment of GRMZM2G039934 between Huangzaosi and LV28. There were total 19 sequence polymorphisms between Huangzaosi and LV28, among which 17 sequence polymorphisms were located in the coding region; the others are located in the introns. (TIF $218 \mathrm{~kb}$ )

\section{Abbreviations}

10KL: 10-kernel length; 10-KW: 10-kernel width; AP: association mapping pane B73 RefGen_v2: B73 reference genome v2.0 assembly; BR: brassinosteroid; HKW: hundred kernel weight; HZS: Huangzaosi; InDel: insert and deletion; K: kinship; MLM: mixed linear model; PCA: principal component analysis; PCR: polymerase chain reaction; Q: population structure; QTL: quantitative trait loci; RIL: recombinant inbred line; SNP: single-nucleotide polymorphisms; SSR: simple sequence repeat.

\section{Competing interests}

The authors declare that they have no competing interests.

\section{Authors' contributions}

TW conceived and designed the study and carried out all the experiments. YuL participated in the design and coordination of the study and provided critical reading of the manuscript. LC performed all the experiments, analysed the related data and wrote the manuscript. YoL participated in the design of the study, assisted in the statistical analysis and provided critical reading of the manuscript. CL provided the RIL population data and assisted in the QTL mapping analysis. XW provided the related data for the regional association-mapping panel. WQ, XL and FJ assisted in the fieldwork and the evaluation of the phenotype. $X Z$ assisted in the candidate gene association mapping. DZ assisted in the sequence analysis. YuS and YaS assisted in the field management and prepared the related reagents. All authors have read and approved the final version of the manuscript.

\section{Acknowledgements}

This work was partly supported by grants provided by the National Natural Science Foundation of China (91335206), the Ministry of Science and Technology of China (2011CB100105, 2014CB138200), and CAAS (Innovation Program). We are grateful to Professor Jinsheng Lai of China Agricultural University for sharing their re-sequence information for the marker development in this study.

\section{Funding}

This work was partly supported by grants provided by the National Natural Science Foundation of China (91335206), the Ministry of Science and Technology of China (2011CB100105, 2014CB138200), and CAAS (Innovation Program).
Received: 13 October 2015 Accepted: 6 April 2016

Published online: 12 April 2016

\section{References}

1. Salvi S, Tuberosa R, Chiapparino E, Maccaferri M, Veillet S, Beuningen LV, Isaac P, Edwards K, Phillips RL. Toward positional cloning of Vgt1, a QTL controlling the transition from the vegetative to the reproductive phase in maize. Plant Mol Biol. 2002;48:601-13.

2. Hung HY, Shannon LM, Tian F, Bradbury PJ, Chen C, Flint-Garcia SA, McMullen MD, Ware D, Buckler ES, Doebley JF et al. ZmCCT and the genetic basis of daylength adaptation underlying the postdomestication spread of maize. Proc Natl Acad Sci U S A. 2012;109(28):E1913-21.

3. Yang Q, Li Z, Li W, Ku L, Wang C, Ye J, Li K, Yang N, Li Y, Zhong T et al. CACTA-like transposable element in ZmCCT attenuated photoperiod sensitivity and accelerated the postdomestication spread of maize. Proc Natl Acad Sci U S A. 2013;110(42):16969-74.

4. Gallavotti A, Barazesh S, Malcomber S, Hall D, Jackson D, Schmidt RJ, McSteen P. sparse inflorescencel encodes a monocot-specific YUCCA-like gene required for vegetative and reproductive development in maize. Proc Natl Acad Sci U S A. 2008;105(39):15196-201.

5. Zhang J, Ku LX, Han ZP, Guo SL, Liu HJ, Zhang ZZ, Cao LR, Cui XJ, Chen YH. The ZmCLA4 gene in the qLA4-1 QTL controls leaf angle in maize (Zea mays L.). J Exp Bot. 2014;65(17):5063-76.

6. Taguchi-Shiobara F, Yuan Z, Hake S, Jackson D. The fasciated ear2 gene encodes a leucine-rich repeat receptor-like protein that regulates shoot meristem proliferation in maize. Genes Dev. 2001:15(12):2755-66.

7. Bommert P, Nagasawa NS, Jackson D. Quantitative variation in maize kernel row number is controlled by the FASCIATED EAR2 locus. Nat Genet. 2013; 45(3):334-7.

8. Wang H, Nussbaum-Wagler T, Li B, Zhao Q, Vigouroux Y, Faller M, BombliesYant K, Lukens L, Doebley J. The origin of the naked grains of maize. Nature. 2005:436(6):714-9.

9. Martin A, Lee J, Kichey T, Gerentes D, Zivy M, Tatout C, Dubois F, Balliau T, Valot B, Davanture M, Tercé-Laforgue T, Quilleré I, Coque M, Gallais A, Gonzalez-Moro MB, Bethencourt L, Habash DZ, Lea PJ, Charcosset A, Perez P, Murigneux A, Sakakibara H, Edwards KJ, Hirel B. Two cytosolic glutamine synthetase isoforms of maize are specifically involved in the control of grain production. Plant Cell. 2006;18(11):3252-74.

10. Carlson S, Chourey P. A re-evaluation of the relative roles of two invertases, INCW2 and IVR1, in developing maize kernels and other tissues. Plant Physiol. 1999;121(3):1025-35.

11. Maitz M, Santandrea G, Zhang Z, Lal S, Hannah LC, Salamini F, Thompson RD. rgf1, a mutation reducing grain filling in maize through effects on basal endosperm and pedicel development. Plant J. 2000;23(1):29-42.

12. Thevenot C. QTLs for enzyme activities and soluble carbohydrates involved in starch accumulation during grain filling in maize. J Exp Bot. 2005;56(413): 945-58.

13. Lid SE, Gruis D, Jung R, Lorentzen JA, Ananiev E, Chamberlin M, Niu X Meeley R, Nichols S, Olsen OA. The defective kernel 1 (dek1) gene required for aleurone cell development in the endosperm of maize grains encodes a membrane protein of the calpain gene superfamily. Proc Natl Acad Sci U S A. 2002:99(8):5460-5.

14. Zhang Z, Liu Z, Hu Y, Li W, Fu Z, Ding D, Li H, Qiao M, Tang J. QTL analysis of kernel-related traits in maize using an immortalized $F_{2}$ population. PLoS One. 2014;9(2), e89645

15. Xu M, Jiang L, Ge M, Zhao H, Zhang T. Analysis of heterosis and quantitative trait loci for kernel shape related traits using triple testcross population in maize. PLoS One. 2015;10(4), e0124779.

16. Liu Y, Wang L, Sun C, Zhang Z, Zheng Y, Qiu F. Genetic analysis and major QTL detection for maize kernel size and weight in multi-environments. Theor Appl Genet. 2014;127(5):1019-37.

17. Peng B, Wang Y, Li Y-X, Liu C, Liu Z-Z, Wang D, an W-W, Zhang Y, Sun B-C, Shi Y-S, Song Yan-Chun, Wang Tian-Yu, LI Yu. QTL analysis for yield components and kernel-related traits in maize under different water regimes. Acta Agron Sin. 2010;36(11):1832-42.

18. Liu R, Jia H, Cao X, Huang J, Li F, Tao Y, Qiu F, Zheng Y, Zhang Z. Fine mapping and candidate gene prediction of a pleiotropic quantitative trait locus for yield-related trait in Zea mays. PLoS One. 2012;7(11), e49836

19. Feuillet C, Eversole K. Solving the maze. Science. 2009;326(5956):1071-2. 
20. Gaut BS, Le Thierry d'Ennequin M, Peek AS, Sawkins MC. Maize as a model for the evolution of plant nuclear genomes. Proc Natl Acad Sci U S A. 2000; 97:7008-15.

21. Lai J. Gene loss and movement in the maize genome. Genome Res. 2004; 14(10a):1924-31.

22. Bortiri $E$, Jackson D, Hake S. Advances in maize genomics: the emergence of positional cloning. Curr Opin Plant Biol. 2006;9(2):164-71.

23. Buckler ES, Gaut BS, McMullen MD. Molecular and functional diversity of maize. Curr Opin Plant Biol. 2006;9(2):172-6.

24. Wallace JG, Larsson SJ, Buckler ES. Entering the second century of maize quantitative genetics. Heredity. 2014;112(1):30-8.

25. Buckler ES, Holland JB, Bradbury PJ, Acharya CB, Brown PJ, Browne C, Ersoz E, Flint-Garcia S, Garcia A, Glaubitz JC, Goodman MM, Harjes C, Guill K, Kroon DE, Larsson S, Lepak NK, Li H, Mitchell SE, Pressoir G, Peiffer JA, Rosas MO, Rocheford TR, Romay MC, Romero S, Salvo S, Sanchez Villeda H, da Silva HS, Sun Q, Tian F, Upadyayula N, Ware D, Yates H, Yu J, Zhang Z, Kresovich S, McMullen MD. The genetic architecture of maize flowering time. Science. 2009; 325(5941):714-8

26. Tian F, Bradbury PJ, Brown PJ, Hung H, Sun Q, Flint-Garcia S, Rocheford TR, McMullen MD, Holland JB, Buckler ES. Genome-wide association study of leaf architecture in the maize nested association mapping population. Nat Genet. 2011;43(2):159-62.

27. Kump KL, Bradbury PJ, Wisser RJ, Buckler ES, Belcher AR, Oropeza-Rosas MA, Zwonitzer JC, Kresovich S, McMullen MD, Ware D, Balint-Kurti PJ, Holland JB. Genome-wide association study of quantitative resistance to southern leaf blight in the maize nested association mapping population. Nat Genet. 2011;43(2):163-8.

28. Peiffer JA, Flint-Garcia SA, Leon ND, McMullen MD, Kaeppler SM, Buckler ES. The genetic architecture of maize stalk strength. PLoS One. 2013;8(6), e67066.

29. Peiffer JA, Romay MC, Gore MA, Flint-Garcia SA, Zhang Z, Millard MJ, Gardner CAC, McMullen MD, Holland JB, Bradbury PJ, Buckler ES. The genetic architecture of maize height. Genetics. 2014;196(4):1337-56.

30. Doebley J, Bacigalupo A, Stec A. Inheritance of kernel weight in two maizeteosinte hybrid populations implications for crop evolution. Heredity. 1994; 85(3):191-5

31. Ajnone-Marsan P, Monfredini G, Ludwig WF, Melchinger AE, Franceschini $P$, Pagnotto G, Motto M. In an elite cross of maize a major quantitative trait locus controls one-fourth of the genetic variation for grain yield. Theor Appl Genet. 1994;90(3-4):415-24.

32. Austin DF, Lee $M$. Comparative mapping in $F_{2: 3}$ and $F_{6: 7}$ generations of quantitative trait loci for grain yield and yield components in maize. Theor Appl Genet. 1996;92:817-26.

33. Li C, Li Y, Sun B, Peng B, Cheng L, Liu Z, Yang Z, Li Q, Tan W, Zhang Y, Wang D, Shi Y, Song Y, Wang T, Li Y. Quantitative trait loci mapping for yield components and kernel-related traits in multiple connected RIL populations in maize. Euphytica. 2013;193(3):303-16.

34. Yu LI, Wang T-Y. Germplasm base of maize breeding in China and formation of foundation parents. Journal of Maize Sciences. 2010;18(5):8.

35. Xin M, Yang R, Li G, Chen H, Laurie J, Ma C, Wang D, Yao Y, Larkins BA, Sun Q, Yadegari R, Wang $X, N i$ Z. Dynamic expression of imprinted genes associates with maternally controlled nutrient allocation during maize endosperm development. Plant Cell. 2013; 25(9):3212-27.

36. Zhan J, Thakare D, Ma C, Lloyd A, Nixon NM, Arakaki AM, Burnett WJ, Logan KO, Wang D, Wang X, Drews GN, Yadegari R. RNA sequencing of lasercapture microdissected compartments of the maize kernel identifies regulatory modules associated with endosperm cell differentiation. Plant Cell. 2015;27(3):513-31.

37. Veldboom LR, Lee $M$, Woodman WL. Molecular marker-facilitated studies in an elite maize population: I. Linkage analysis and determination of QTL for morphological traits. Theor Appl Genet. 1994;88(1):7-16.

38. Peng B, Li Y, Wang Y, Liu C, Liu Z, Tan W, Zhang Y, Wang D, Shi Y, Sun B, Wang T, Li Y. QTL analysis for yield components and kernelrelated traits in maize across multi-environments. Theor Appl Genet. 2011;122(7):1305-20

39. Li JZ, Zhang ZW, Li YL, Wang QL, Zhou YG. QTL consistency and metaanalysis for grain yield components in three generations in maize. Theor Appl Genet. 2011;122(4):771-82.

40. Wang $Y$, Huang Z, Deng D, Ding H, Zhang R, Wang S, Bian Y, Yin Z, Xu X. Meta-analysis combined with syntenic metaQTL mining dissects candidate loci for maize yield. Mol Breed. 2013;31(3):601-14.
41. Prado SA, Lopez CG, Senior ML, Borras L. The genetic architecture of maize (Zea mays L.) kernel weight determination. G3. 2014:4(9):1611-21.

42. Wu X, Li Y, Shi Y, Song Y, Wang T, Huang Y, Li Y. Fine genetic characterization of elite maize germplasm using high-throughput SNP genotyping. Theor Appl Genet. 2014;127(3):621-31.

43. Moreau L, Charcosset A, Gallais A. Use of trial clustering to study OTL x environment effects for grain yield and related traits in maize. Theor Appl Genet. 2004;110(1):92-105.

44. Fan C, Xing Y, Mao H, Lu T, Han B, Xu C, Li X, Zhang Q. GS3, a major QTL for grain length and weight and minor QTL for grain width and thickness in rice, encodes a putative transmembrane protein. Theor Appl Genet. 2006; 112(6):1164-71.

45. Weng J, Gu S, Wan X, Gao H, Guo T, Su N, Lei C, Zhang X, Cheng Z, Guo X, Wang J, Jiang L, Zhai H, Wan J. Isolation and initial characterization of GW5, a major QTL associated with rice grain width and weight. Cell Res. 2008; 18(12):1199-209.

46. Lu M, Xie C-X, Li X-H, Hao Z-F, Li M-S, Weng J-F, Zhang D-G, Bai L, Zhang S-H. Mapping of quantitative trait loci for kernel row number in maize across seven environments. Mol Breed. 2010;28(2):143-52.

47. Kesavan M, Song JT, Seo HS. Seed size: a priority trait in cereal crops. Physio Plant. 2013;147(2):113-20.

48. Li C, Li Y, Shi Y, Song Y, Zhang D, Buckler ES, Zhang Z, Wang T, Li Y. Genetic control of the leaf angle and leaf orientation value as revealed by ultra-high density maps in three connected maize populations. PLoS One. 2015;10(3), e0121624.

49. Courtial A, Thomas J, Reymond M, Mechin V, Grima-Pettenati J, Barriere Y. Targeted linkage map densification to improve cell wall related QTL detection and interpretation in maize. Theor Appl Genet. 2013;126(5): 1151-65.

50. Song XJ, Huang W, Shi M, Zhu MZ, Lin HX. A QTL for rice grain width and weight encodes a previously unknown RING-type E3 ubiquitin ligase. Nat Genet. 2007:39(5):623-30.

51. Morinaka YST, Inukai Y, Agetsuma M, Kitano H, Ashikari M, Matsuoka M. Morphological alteration caused by brassinosteroid insensitivity increases the biomass and grain production of rice. Plant Physiol. 2006;141(3):924-31.

52. Tanaka KAT, Yoshida S, Nakamura Y, Matsuo T, Okamoto S. Brassinosteroid homeostasis in Arabidopsis is ensured by feedback expressions of multiple genes involved in its metabolism. Plant Physiol. 2005;138(2):1117-25.

53. Wu Y, Fu Y, Zhao S, Gu P, Zhu Z, Sun C, Tan L. CLUSTERED PRIMARY BRANCH 1, a new allele of DWARF11, controls panicle architecture and seed size in rice. Plant Biotechnol J. 2016;14(1):377-86.

54. Hong Z. The rice brassinosteroid-deficient dwarf2 mutant, defective in the rice homolog of Arabidopsis DIMINUTO/DWARF1, is rescued by the endogenously accumulated alternative bioactive brassinosteroid, dolichosterone. Plant Cell. 2005:17(8):2243-54.

55. Tanabe S. A novel cytochrome P450 is implicated in brassinosteroid biosynthesis via the characterization of a rice dwarf mutant, dwarf11, with reduced seed length. Plant Cell. 2005;17(3):776-90.

56. Wu CYTA, Radhakrishnan P, Kwok SF, Harris S, Zhang K, Wang J, Wan J, Zhai H, Takatsuto S, Matsumoto S, Fujioka S, Feldmann KA, Pennell RI. Brassinosteroids regulate grain filling in rice. Plant Cell. 2008:20(8):2130-45.

57. Li Y, Ma X, Wang T, Li Y, Liu C, Liu Z, Sun B, Shi Y, Song Y, Carlone M et al. Increasing maize productivity in China by planting hybrids with germplasm that responds favorably to higher planting densities. Crop Sci. 2011;51(6): 2391.

58. Zuo W, Chao Q, Zhang N, Ye J, Tan G, Li B, Xing Y, Zhang B, Liu H, Fengler $\mathrm{KA}$ et al. A maize wall-associated kinase confers quantitative resistance to head smut. Nat Genet. 2014;47(2):151-7.

59. Zhao X, Tan G, Xing Y, Wei L, Chao Q, Zuo W, Lübberstedt T, Xu M. Markerassisted introgression of $\mathrm{qHSR} 1$ to improve maize resistance to head smut. Mol Breed. 2012;30(2):1077-88.

60. Jiao Y, Zhao H, Ren L, Song W, Zeng B, Guo J, Wang B, Liu Z, Chen J, Li W et al. Genome-wide genetic changes during modern breeding of maize. Nat Genet. 2012:44(7):812-5.

61. Ronald PC, Chen DH. A rapid DNA minipreparation method suitable for AFLP and other PCR applications. Plant Mol Biol Report. 1999:17:4.

62. Lincoln SE, Daly MJ, Lander ES. Constructing genetic maps with MAPMAKER_EXP 3.0. In: Technical report. Cambridge, MA: Whitehead Institute; 1992. p. 86.

63. Li H, Ye G, Wang J. A modified algorithm for the improvement of composite interval mapping. Genetics. 2006;175(1):361-74. 
64. Wang J, Wan X, Crossa J, Crouch J, Weng J, Zhai H, Wan J. QTL mapping of grain length in rice (Oryza sativa L.) using chromosome segment substitution lines. Genet Res. 2006;88(02):93-104.

65. Yu J, Pressoir G, Briggs WH, Vroh Bi I, Yamasaki M, Doebley JF, McMullen MD, Gaut BS, Nielsen DM, Holland JB et al. A unified mixed-model method for association mapping that accounts for multiple levels of relatedness. Nat Genet. 2005;38(2):203-8.

66. Bradbury PJ, Zhang Z, Kroon DE, Casstevens TM, Ramdoss Y, Buckler ES. TASSEL: software for association mapping of complex traits in diverse samples. Bioinformatics. 2007;23(19):2633-5.

67. Barrett JC, Fry B, Maller J, Daly MJ. Haploview: analysis and visualization of LD and haplotype maps. Bioinformatics. 2004;21(2):263-5.

68. Hall TA. BioEdit: a user-friendly biological sequence alignment editor and analysis program for Windows 95_98_NT. Nucleic Acids Symp Ser. 1999;41:95-8

Submit your next manuscript to BioMed Central and we will help you at every step:

- We accept pre-submission inquiries

- Our selector tool helps you to find the most relevant journal

- We provide round the clock customer support

- Convenient online submission

- Thorough peer review

- Inclusion in PubMed and all major indexing services

- Maximum visibility for your research

Submit your manuscript at www.biomedcentral.com/submit 